\title{
Isolated crohn's disease of esophagus: a rarest of rare presentation
}

\begin{abstract}
We present a case of a 19-year -old female complaining of dysphagia for over 2 months. Because of her age and the rarity of Crohn's disease in this particular age group, the patient went several months without the right diagnosis and treatment. The diagnosis of Crohn's disease was finally achieved via upper endoscopy with the patient having resolution of symptoms after correct treatment was started. This is the first case of esophageal Crohn's disease in a young female found in literature to date.
\end{abstract}

Keywords: dysphagia, nausea, abdominal pain, tums, stool antigen
Volume 9 Issue 5 - 2018

\author{
Arvind K Mathur, Lysa Ho \\ Department of Internal Medicine, Hemet Valley Medical Center, \\ USA
}

Correspondence: Arvind K Mathur, Department of Internal Medicine, Hemet Valley Medical Center, USA

Email jaipurse@gmail.com

Received:July 15, 2018| Published: September 18, 2018
Abbreviations: CD, Crohn's disease; GI, gastrointestinal; EGD, esophagoastroduodenoscopy; PO, pantoprazole orally

\section{Case report}

A previously healthy 19-year-old obese female presented with complaints of persistent dysphagia for several months. Symptoms were more prominent when laying flat and eating solid food. She had occasionally tried over-the-counter "tums" only with minimal relief. She denied any headaches, visual disturbances, chest pain, abdominal pain, nausea, vomiting, diarrhea or constipation. She denied any tobacco use and any alcohol consumption. She went to see a primary care physician, who tested her for Helicobacter pylori via serology and stool antigen, which both came back negative. She was then placed on an empiric trial of pantoprazole $40 \mathrm{mg}$ orally (PO) daily for 30 days, later extended to $40 \mathrm{mg}$ PO twice daily for another 30 days, with minimal relief. Though uncertain, she was still given the diagnosis of gastro-esophageal reflux disease, which she was told was exacerbated by her excess weight. She was advised to lose weight, decrease spicy food intake, and sleep with head of the bed elevated. She complied with all the recommendations, including diet changes and exercise, and was successful in reducing her weight of 220 pounds to 180 pounds in 4 months. Her symptoms, however, still persisted. Pantoprazole was then changed to dexlansoprazole $30 \mathrm{mg}$ PO daily for 30 days and then later increased to $60 \mathrm{mg}$ PO daily for another 4 weeks. Her symptoms did not improve significantly after 4 months of proton pump inhibitor therapy. Routine labs including complete blood count, comprehensive metabolic panel, serum pre-albumin, sedimentation rate and C-reactive protein were performed and were entirely normal. She was then referred to a gastroenterologist, who performed an upper endoscopy.

The upper endoscopy showed erythema and a full circumferential superficially ulcerated lesion of the mucosa, with cobblestone appearance in the upper third of the esophagus, $22 \mathrm{~cm}$ to $24 \mathrm{~cm}$ from incisors. No narrowing of the lumen was noted. Biopsy specimens were taken. Remaining esophagus was normal. Abdominal computed tomography scan with and without IV and oral contrast was normal. A colonoscopy and a capsule endoscopy were performed and were negative for any lesions on gross visualization and pathology. The biopsy results from the esophageal samples were consistent with acute and chronic inflammation, and non-caseating granulomas. Serum Antineutrophil cytoplasmic antibodies and anti-Saccharomyces cerevisiae antibodies were negative. Patient was started on $40 \mathrm{mg}$ orally daily of prednisone and a 6-week course of oral steroids, resulting in near complete resolution of her symptoms.

\section{Discussion}

Crohn's disease (CD) is a chronic inflammatory disease of the gastrointestinal (GI) tract, characterized by discontinuous segmental, granulomatous, and trans-mural inflammation with episodic relapse and remission, leading to complications of strictures, fistulas, or abscesses. ${ }^{1,2}$ Although CD can occur anywhere from the oral

cavity to the anus, the most usual occurrences are in the ileum and colon. ${ }^{3}$ The esophagus is the least common location for CD, and it was first described by Franklin and Taylor in $1950 .{ }^{4}$ In the recent quarter century, prevalence of esophageal $\mathrm{CD}$ has gradually increased ranging from $0.2 \%$ in a couple of retrospective observational series to $11 \%$ in another. Although 14 years apart, Decker et al. ${ }^{5}$ and De Felice et al. ${ }^{6}$ both found no more than a $0.2 \%$ prevalence of CD with esophageal involvement in a patient population of 9900 and 12367 respectively, while D'Haens et al., ${ }^{7}$ demonstrated an $11 \%$ prevalence in 124 patients. All of the series had concurrent CD elsewhere on the GI tract, except De Felicia and colleagues designated 3 of 24 cases as having remote esophageal $\mathrm{CD}$.

Therefore, isolated esophageal $\mathrm{CD}$ is an even more rare finding that has only been previously described in the aforementioned study among scarce case reports. Ours is probably the first case reported in literature of isolated Crohn's disease diagnosed during teenage years. This is in part due to low threshold for esophagogastroduodenoscopy (EGD) for upper GI symptoms in undiagnosed $\mathrm{CD}$ as well as the diagnostic challenges surrounding this disease manifestation. Mild esophageal CD can present as erosive esophagitis, with more advanced disease showing ulcers and/or strictures, which are common features of secondary esophagitis. ${ }^{6}$ These comprise of infectious esophagitis (herpes simplex virus, cytomegalovirus, mycobacteria, syphilis, histoplasmosis), gastro-esophageal reflux disease, caustic agent ingestion, sarcoidosis, medication induced and radiation induced esophagitis, Behcet's disease, malignancy and esophageal lichen planus. ${ }^{6,8-10}$ 
A majority of patients presents for evaluation of progressive dysphagia, odynophagia, or worsening reflux. ${ }^{6,11}$ In a series by De Felice et al., ${ }^{6}$ out of the 24 patients who had esophageal CD, dysphasia was present in $54 \%$ of the patients, odynophagia in $33 \%$, epigastric pain in $33 \%$, heartburn in $25 \%$, and nonspecific chest pain in $13 \%$. In addition, one patient had failure to thrive and three patients underwent EGD for evaluation of weight loss and decreased appetite. It is important to note that any patient with an existing diagnosis of CD warrants EGD with biopsy even for minor upper GI complaints. ${ }^{11}$

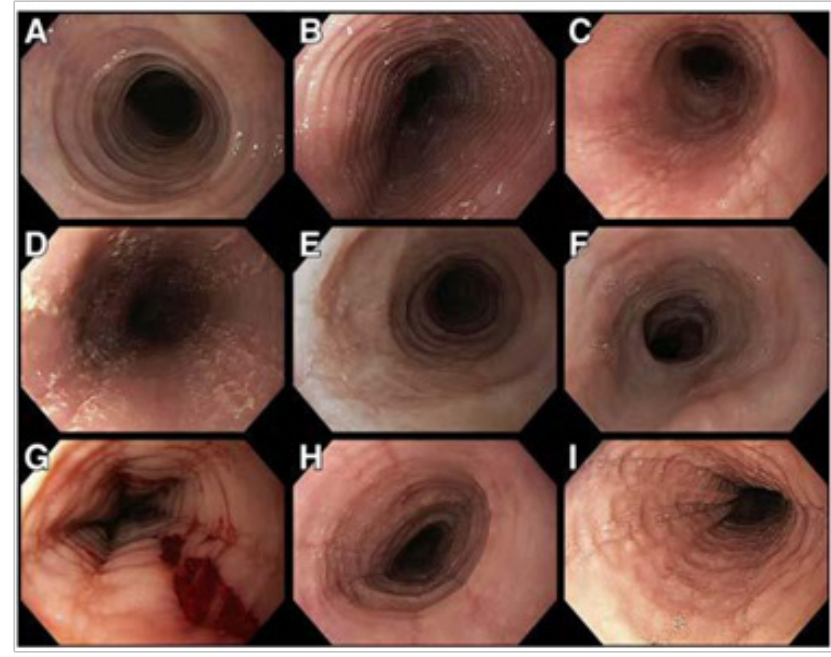

Figure I EGD images of Crohn's disease of esophagus.

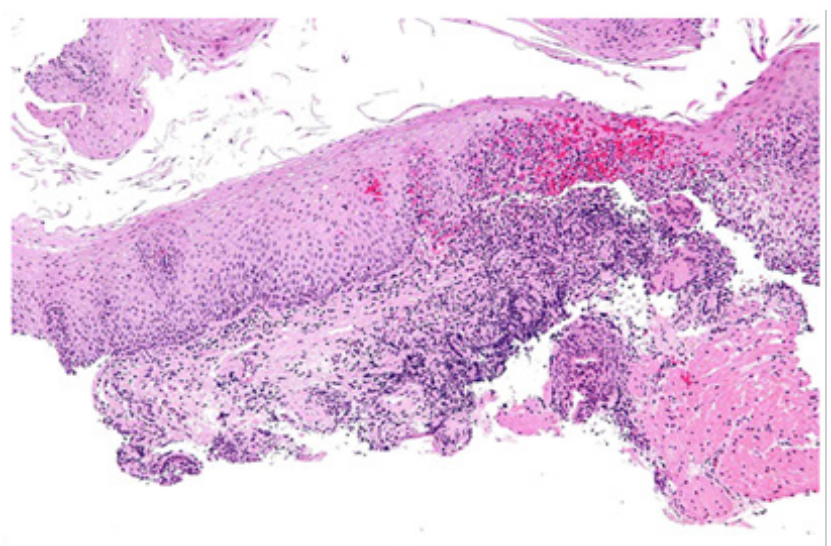

Figure 2 Esophagel crohn's disease slide image.

Endoscopy with biopsy is always diagnostic for any type of CD. For esophageal CD, the most common site of occurrence is mid or distal esophagus, accounting for $29 \%$ of cases individually. Furthermore, $75 \%$ of the study population had inflammatory esophageal $\mathrm{CD}$, marked by superficial ulcerations, erythema with and without erosions, deep ulcerations and pseudo polyps on endoscopy. Seventeen percent were found to have esophageal strictures and $8 \%$ had fistulizing disease. ${ }^{6}$ In a more recent study in $2015,30 \%$ to $40 \%$ of patients had apththous ulcerations, mucosal nodules or the typical CD's cobblestone appearance. ${ }^{11}$ Additional testing to include antisaccharomyces cerevisiae antibodies, found in $>50 \%$ of CD patients, can be beneficial to further differentiate $\mathrm{CD}$ from ulcerative colitis. ${ }^{3}$

Although, histology in previous serial studies has shown nonspecific findings; yet, it is still imperative for a definitive diagnosis of CD. These usually contain evidence of chronic inflammation with plasma cells and lymphocytes and infrequently, non-caseating granuloma formation and acute inflammation, showing predominantly polymorphonuclear neutrophils. One study presented 7 of $27(25 \%)$ in a pediatric patient group, and another with 5 of 24 (20\%) from the adult group with non-caseating granuloma. ${ }^{6,12}$ In a case report in Korea, histopathological findings of a patient with esophagogastric CD revealed non-caseating granuloma in the ulcer bed of the esophagus, as it was present in the lamina propria of the stomach with positive CD68, without evidence of viral, bacterial or fungal infection. ${ }^{13}$

The management of esophageal $\mathrm{CD}$ can vary depending on disease severity. Previous studies have demonstrated that steroids, oral prednisone or prednisolone up to $60 \mathrm{mg}$ per day, were effective in controlling inflammation with the addition of acid suppression therapy for upper GI symptoms. ${ }^{6,9,12}$ In one of the largest published series of its time, the use of systemic corticosteroids after 2 to 4 weeks resulted in complete resolution of symptoms in more than $50 \%$ of patients. ${ }^{6,11,14}$ Acid suppression with either proton pump inhibitor or $\mathrm{H} 2$ blocker were initiated in patients with combined esophageal and ileo-colonic $\mathrm{CD}$, mainly due to patients noticing heartburn and nausea after lower GI symptoms were addressed with systemic steroids and nutrition. ${ }^{12}$ Swallowed aerosolized budesonide, as a novel adjuvant topical treatment, in addition to conventional therapy has been reported as successful. ${ }^{6,11,15}$ For those who are steroid dependent or steroid resistant, several studies have proven immune modifiers as efficacious. In the study at the Mayo Clinic by Decker et al., ${ }^{5} 11$ patients received azathioprine, ${ }^{6}$ mercaptopurine, or cyclosporine, and more than $50 \%$ exhibited significant improvement. Similarly, infliximab, a TNF $\alpha$ inhibitor, was effective in preventing complications of strictures or fistula formation in refractory patients. ${ }^{16}$ However, in a case of lifethreatening esophageal $\mathrm{CD}$ in which resulted in the patient losing $14 \%$ of his body weight, anti-TNF $\alpha$ injection was started as first line therapy, with the patient having complete symptom resolution after his $2^{\text {nd }}$ dose, and gaining back his original weight after the $3^{\text {rd }} .^{13}$ Nonetheless, there have yet to exist controlled trials verifying antiTNF $\alpha$ 's efficacy as first line therapy; therefore, the decision to initiate treatment should be proceeded with caution.

Complications such as stricture and fistula formations were successfully treated with a combination of biologic therapy, immunomodulators, and endoscopic therapy, including stricture dilation for esophageal strictures, with and without steroid injections; however, endoscopic therapy for fistulizing esophageal $\mathrm{CD}$ did not remain consistently effective. ${ }^{6}$ Patients would then continue to have recurrent mediastinal inflammation, abscesses, or pneumomediastium. Surgical intervention is almost always required for fistula formation, albeit in a case report, complete closure of an esophagobronchial fistula was achieved via infliximab injections only. ${ }^{17}$ Surgery was also prevented in a patient with esophagogastric fistula with the induction of infliximab. ${ }^{10}$ Nevertheless, esophagectomy was the typical surgical procedure performed for complicated fistula or obstruction, as described in several literature reviews. ${ }^{2,5,6,8,11}$ Wang et $\mathrm{al}^{2}{ }^{2}$ in their isolated esophageal CD with esophago-mediastinal fistula, esophagectomy was performed with gastric pull-through with positive response. $^{2}$

In conclusion, esophageal $\mathrm{CD}$ continues to be a challenging diagnosis. Given the possibility of isolated esophageal $\mathrm{CD}$, anyone with upper GI symptoms refractory to acid suppression therapy, antibiotics, and are less than 50years of age, EGD should be considered. In addition, those with previously diagnosed $\mathrm{CD}$ presenting with 
upper GI symptoms should always prompt a new or repeat upper endoscopy examination. The combination of systemic steroids and acid suppression is the mainstay of treatment. Immunomodifiers, biologics, and/or endoscopic therapy are recommended in refractory CD. Surgery is only recommended in complicated fistulizing or obstructing disease. It is imperative to recognize esophageal involvement early and start medical therapy, as active disease prolongation may lead to devastating consequences.

\section{Acknowledgements}

None.

\section{Conflict of interest}

The author declares no conflict of interest.

\section{References}

1. Colombel JF, Sandborn WJ, Reinisch W, et al. Infliximab, Azathioprine, or combination therapy for crohn's disease. $N$ Engl $J$ Med. 2010;362(15):1383-1395.

2. Wang W, Ni Y, Ke C, et al. Isolated Crohn's disease of the esophagus with esophago-mediastinal fistula formation. World J Surg Oncol. 2012;10:208.

3. Podolsky DK. Inflammatory bowel disease. $N$ Engl $J$ Med. 2002;347(6):417-429.

4. Franklin RH, Taylor S. Nonspecific granulomatous (regional) esophagitis. J Thorac Surg. 1950;19(2):292-297.

5. Decker GA, Loftus EVJ, Pasha TM, et al. Crohn's disease of the esophagus: clinical features and outcomes. Inflamm Bowel Dis. 2001;7(2):113-119.

6. De Felice KM, Katzka DA, Raffals LE. Crohn's disease of the esophagus: Clinical features and treatment outcomes in the biologic era. Inflamm Bowel Dis. 2015;21(9):2106-2113.
7. D'Haens G, Rutgeerts P, Geboes K, et al. The natural history of esophageal Crohn's disease: three patterns of evolution. Gastrointest Endosc. 1994;40(3):296-300.

8. Reynolds HL, Stellato TA. Crohn's disease of the foregut. Surg Clin North Am. 2001;81(1):117-132.

9. Kasarala G, Harvin G, Durrett S. Adult-onset esophageal Crohn's disease. ACG Case Reports J. 2016;3(4):e139.

10. Heller T, James SP, Drachenberg T, et al. Treatment of severe esophageal Crohn's disease with Infliximab. Infect Dis Soc Am. 1999;5(4):279-282.

11. Davis KG. Crohn's disease of the foregut. Surg Clin North Am. 2015;95(6):1183-1193.

12. Ramaswamy K, Jacobson K, Jevon G, et al. Esophageal Crohn disease in children: a clinical spectrum. J Pediatr Gastroenterol Nutr. 2003;36(4):454-458.

13. So H, Park BH, Jang K, et al. Esophagogastric Crohn's disease manifested by life-threatening odynophagia and chest pain: a case report. J Korean Med Sci. 2018;33(4):e30.

14. D'Haens G, Rutgeerts P, Geboes K, et al. The natural history of esophageal Crohn's disease: three patterns of evolution. Gastrointest Endosc. 1994;40(3):296-300.

15.Zezos P, Kouklakis G, Oikonomou A, et al. Esophageal Crohn's disease treated "topically" with swallowed aerosolized budesonide. Case Rep Med. 2010;2010:4.

16. Pantanowitz L, Gelrud A, Apstein M, et al. Crohn's disease of the esophagus. Ear, Nose Throat J. 2004;83(6):420-423.

17. Ho IK, Guarino DP, Pertsovskiy Y, et al. Infliximab treatment of an esophagobronchial fistula in a patient with extensive Crohn's disease of the esophagus. J Clin Gastroenterol. 2002;34(4):488-489. 